\title{
Two-Dimensional Binary Honeycomb Layer Formed by Ag and Te on Ag(111)
}

\author{
J. Shah, H. M. Sohail, R. I. G. Uhrberg, and W. Wang*
}

Cite This: J. Phys. Chem. Lett. 2020, 11, 1609-1613

Read Online

ABSTRACT: Inspired by the unique properties of graphene, research efforts have broadened to investigations of various other two-dimensional materials with the aim of exploring their properties for future applications. Our combined experimental and theoretical study confirms the existence of a binary honeycomb structure formed by $\mathrm{Ag}$ and $\mathrm{Te}$ on $\mathrm{Ag}(111)$. Low-energy electron diffraction shows sharp spots which provide evidence of an undistorted AgTe layer. Band structure data obtained by angle-resolved photoelectron spectroscopy are closely reproduced by first-principles calculations, using density functional theory (DFT). This confirms the formation of a honeycomb structure with one $\mathrm{Ag}$ and one $\mathrm{Te}$ atom in the unit cell. In addition, the theoretical band structure reproduces also the finer details of the experimental bands, such as a split of one of the AgTe bands.
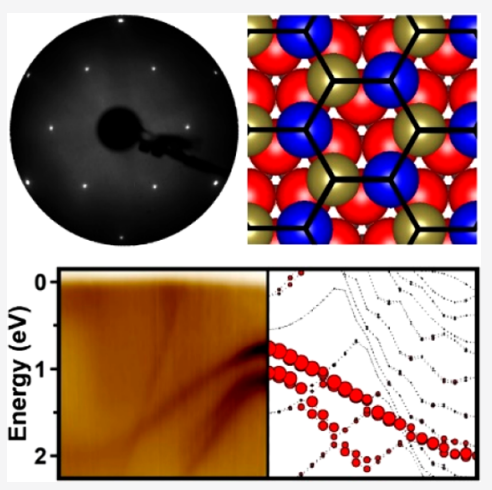

$\mathrm{T}$ he research field of two-dimensional (2D) materials has attracted great attention in recent years. Such materials may have unique properties that are not present in the threedimensional bulk. The search for 2D materials for applications in the next generation of electronic and optoelectronic devices is currently quite intense. Prominent examples are the discovery of graphene ${ }^{1}$ and subsequent investigations of single-element graphene-analogues from group IV, such as silicene, germanene, and stanene. ${ }^{2}$ Another type of $2 \mathrm{D}$ materials consists of high- $Z(Z=$ atomic number $)$ substitutional surface alloys on noble metals. Surface alloying is recognized as a viable way to achieve unique physical and chemical properties not found in the bulk. For example, a giant Rashba-type of spin splitting ${ }^{3}$ was observed for $\mathrm{Ag}_{2} \mathrm{Bi}^{4}{ }^{4}$ while the corresponding replacement of $\mathrm{Ag}$ by the lighter element $\mathrm{Sb}$ resulted in a barely observable split. ${ }^{5}$ Very recently, a $2 \mathrm{D} \mathrm{Sb}$ analogue of graphene, so-called antimonene, was reported to be successfully formed on $\operatorname{Ag}(111)$, with a lattice constant and orientation matching a $(\sqrt{3} \times \sqrt{3}) \mathrm{R} 30^{\circ}$ supercell. ${ }^{6}$ Hence, it is very interesting and worthwhile to examine whether Te, the group VI neighbor of $\mathrm{Sb}$, forms a graphene-like flat 2D honeycomb structure or a surface alloy with spin splitting on $\mathrm{Ag}(111)$. Actually, in this Letter, we report experimental and theoretical results that provide evidence for a combination of the two alternatives. We find that the electronic band structure matches that of a binary AgTe 2D honeycomb alloy structure.

The feasibility of forming binary honeycomb structures has been reported for a $2 \mathrm{D}$ layer of $\mathrm{CuSe}$ on $\mathrm{Cu}(111){ }^{7,8}$ Scanning tunneling microscopy (STM) images presented in ref 8 showed a clear honeycomb structure, and the conclusion that the unit cell consisted of one $\mathrm{Cu}$ and one $\mathrm{Se}$ atom was inferred from a comparison between experimental and calculated band structures. From the striped appearance observed in STM images, it was further concluded that the $\mathrm{CuSe}$ honeycomb structure was uniaxially distorted. This deviation from an ideal honeycomb structure resulted in a splitting of the CuSe spots in the low-energy electron diffraction (LEED) pattern from the CuSe layer. ${ }^{8}$ A honeycomb structure has also been reported for 2D layers of AgTe on $\operatorname{Ag}(111)$ in two recent studies. ${ }^{9,10}$ STM and LEED results from a sample annealed at $720 \mathrm{~K}$, in ref 9, showed different structures denoted as buckled, with ridges and trenches, and striped with uniaxially elongated honeycombs. As in the case of $\mathrm{CuSe}$, the various distortions of the honeycomb layer were clearly revealed by additional diffraction spots in the LEED patterns. In ref 10, STM data from a AgTe sample annealed at $500 \mathrm{~K}$ showed a striped structure resembling that of the striped structure discussed in ref 9. The large size of the AgTe spots in the LEED pattern in ref 10 is consistent with the presence of several unresolved spots, as expected from a distorted honeycomb layer.

In our Letter, we present data from a AgTe layer characterized by sharp LEED spots which is evidence for a well-ordered surface layer without the distortions reported in the previous studies of the binary honeycomb layers. ${ }^{7-10}$ Detailed band structure data obtained by angle-resolved

Received: January 13, 2020

Accepted: February 10, 2020

Published: February 10, 2020 

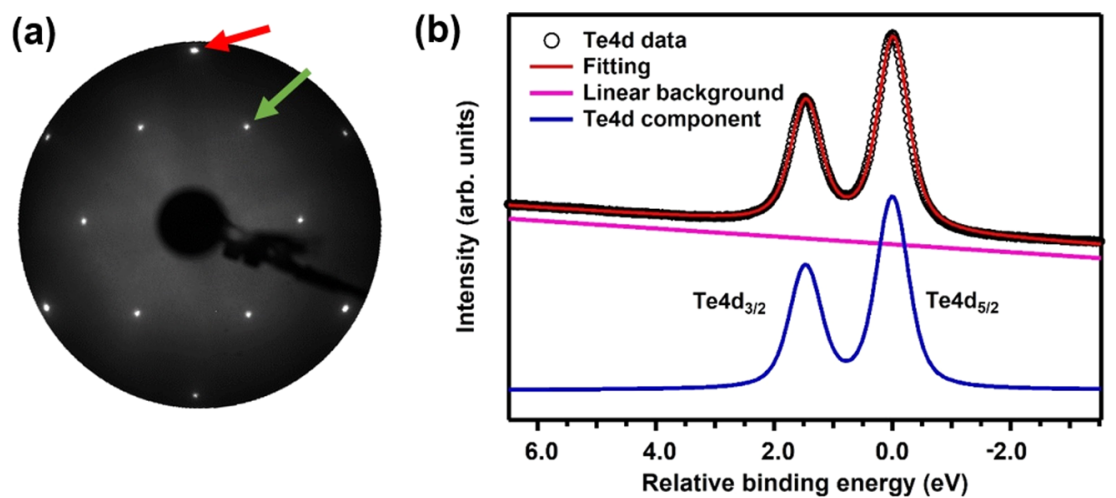

Figure 1. LEED and core-level spectroscopy data. (a) LEED pattern $(78 \mathrm{eV})$ of the $\operatorname{AgTe} / \operatorname{Ag}(111)$ sample showing sharp diffraction spots, indicating a well-ordered structure with a $(\sqrt{3} \times \sqrt{3}) \mathrm{R} 30^{\circ}$ periodicity relative to $\mathrm{Ag}(111)$. One of the $\mathrm{Ag} 1 \times 1$ spots is indicated by a red arrow. The green arrow points at one of the spots from the AgTe layer. (b) Te $4 \mathrm{~d}$ core-level spectrum obtained using a photon energy of $80 \mathrm{eV}$ at normal emission. The black circles are the experimental data, and the fitting curve is the sum of one spin-orbit split component and a linear background. Fitting parameters: spin-orbit split, $1.47 \mathrm{eV}$; branching ratio, 0.636; Gaussian width, $387 \mathrm{meV}$; and Lorentzian width, $373 \mathrm{meV}$. The asymmetry parameter of the Doniach-Sunjić line profile is 0 , which indicates a semiconducting character of the AgTe 2D structure.
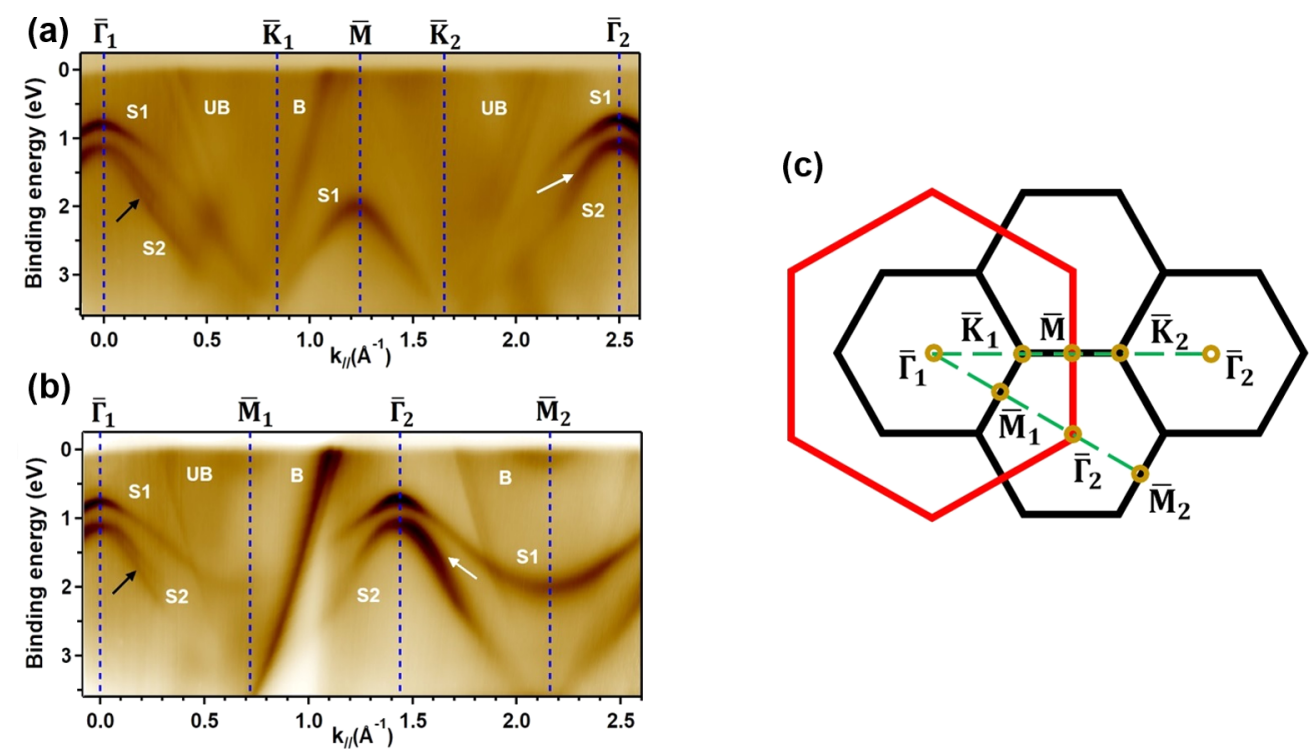

Figure 2. Electronic band structure of $\operatorname{AgTe}$ on $\operatorname{Ag}(111)$ obtained by ARPES. (a) Energy bands mapped along the $\bar{\Gamma} \bar{K} \bar{M} \bar{K} \bar{\Gamma}$ line indicated by the green dashed line in panel c. (b) Energy bands mapped along $\bar{\Gamma} \overline{\mathrm{M}} \bar{\Gamma} \overline{\mathrm{M}}$. The photon energy was $35 \mathrm{eV}$. Two bands are indicated by S1 and S2, respectively, in panels a and $\mathrm{b}$. These bands constitute the $2 \mathrm{D}$ band structure of $\mathrm{AgTe}$ on $\mathrm{Ag}(111)$ along the high-symmetry lines. $\mathrm{B}$ originates from direct transitions involving $\mathrm{Ag}$ sp bulk bands. UB denotes umklapp scattering of B by $\sqrt{3} \times \sqrt{3}$ reciprocal lattice vectors. Black arrows indicate band splittings. White arrows point to places in the band structure, where the splitting is expected, but is less clear in the data. (c) Schematic drawing of SBZs. Red hexagon shows the $1 \times 1$ SBZ of the $\mathrm{Ag}(111)$ surface. Black hexagons represent $\sqrt{3} \times \sqrt{3}$ SBZs of AgTe. Green dashed lines indicate the high-symmetry lines in the SBZs, along which the experimental data were obtained. Several symmetry points are marked by yellow circles.

photoelectron spectroscopy (ARPES) in combination with calculated band structures of various models, based on density functional theory (DFT), is used to verify the formation of a 2D AgTe honeycomb structure. Further, our ARPES data reveal a Rashba type of spin-split in the lower band of the two originating from the AgTe 2D layer.

In order to prepare the AgTe structure, presented in this Letter, a $\operatorname{Ag}(111)$ crystal was cleaned by repeated cycles of sputtering by $\mathrm{Ar}^{+}$ions $(1 \mathrm{keV})$ and annealing at approximately $400{ }^{\circ} \mathrm{C}$ until a sharp $(1 \times 1)$ LEED pattern was obtained. Tellurium was deposited onto the clean $\operatorname{Ag}(111)$ sample at room temperature from a source that was calibrated by a thickness monitor. The data were obtained from a sample prepared by depositing $1 / 3$ monolayer $(\mathrm{ML})$ of Te followed by annealing at approximately $200{ }^{\circ} \mathrm{C}$ for $5 \mathrm{~min}$. After this preparation, LEED showed sharp diffraction spots from the AgTe layer corresponding to a $(\sqrt{ } 3 \times \sqrt{ } 3) \mathrm{R} 30^{\circ}$ periodicity with respect to $\operatorname{Ag}(111)$ (see Figure 1a). The sharpness of the spots indicates an ordered structure with a well-defined periodicity without the type of distortions reported in refs 7-10. Core-level spectroscopy data from the inner shell electrons provide additional information related to the atomic structure. Figure $1 \mathrm{~b}$ shows a high-resolution Te $4 \mathrm{~d}$ core-level spectrum. This spectrum is accurately fitted by just one spinorbit split component plus a linear background. The lack of shifted components indicates that the Te atoms are located at identical positions consistent with a well-ordered structure. 
The band structure of the AgTe layer, probed by ARPES, using a photon energy of $35 \mathrm{eV}$, is presented in Figure $2 \mathrm{a}, \mathrm{b}$. These data reveal a semiconducting band structure in agreement with the symmetric line shape of the Te $4 \mathrm{~d}$ spectrum. The band dispersions follow a $\sqrt{3} \times \sqrt{3}$ periodicity, which is clear from the band mapping along the $\bar{\Gamma} \overline{\mathrm{K}} \overline{\mathrm{M}} \bar{\Gamma}$ and $\bar{\Gamma} \bar{M} \bar{\Gamma}$ paths of the $\sqrt{3} \times \sqrt{3}$ surface Brillouin zone (SBZ), indicated in Figure $2 \mathrm{c}$. From these data, one can conclude that there are two bands separated in energy and momentum space. The upper band, $S 1$, has a maximum at a binding energy $\left(E_{\mathrm{B}}\right)$ of $\sim 0.75 \mathrm{eV}$ at $\bar{\Gamma}_{1}$, with respect to the Fermi level $\left(E_{\mathrm{F}}\right)$. The dispersion of $S 1$ shows a local maximum as well as a local minimum at $\overline{\mathrm{M}}$ along $\overline{\mathrm{K}} \overline{\mathrm{M}} \overline{\mathrm{K}}$ and $\bar{\Gamma} \overline{\mathrm{M}} \bar{\Gamma}$, respectively. This makes $\bar{M}$ a saddle point with a diverging density of states (van Hove singularity). The $\mathrm{S} 2$ band shows a downward dispersion with a maximum $E_{\mathrm{B}}$ of $1.1 \mathrm{eV}$ at the center of the SBZ. In contrast to S1, the S2 band exhibits a small splitting along both $\bar{\Gamma} \bar{M}$ and $\bar{\Gamma} \overline{\mathrm{K}}$, indicated by the black arrows in Figure $2 \mathrm{a}, \mathrm{b}$. This band split is shown more clearly in the ARPES data in Figure 3a,
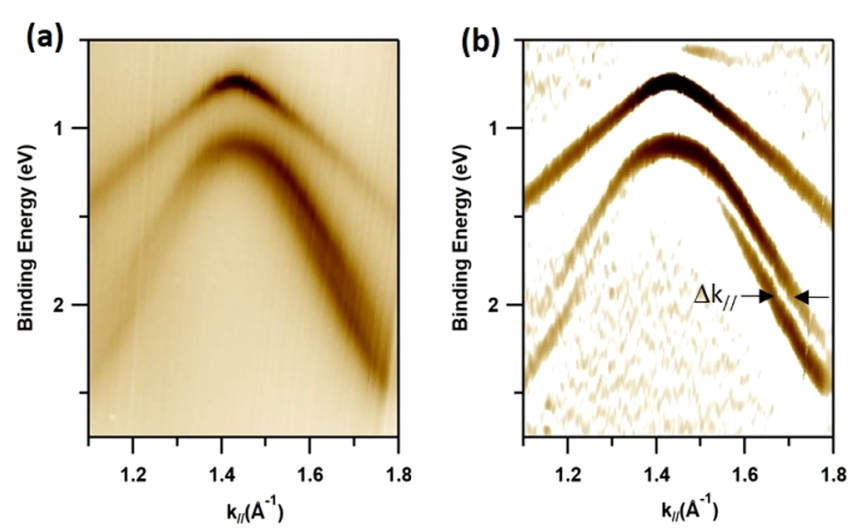

Figure 3. Details of the experimental band structure showing a split of the S2 band. (a) Close up of the dispersions of the S1 and S2 bands in the vicinity of $\bar{\Gamma}_{2}$ along $\bar{\Gamma} \bar{M} \bar{\Gamma}$. The photon energy was $26 \mathrm{eV}$. S2 is split into two sub-bands while no split could be detected for S1. (b) Second derivative along the energy axis of the ARPES data in panel a. The resulting enhanced band structure clearly shows the splitting of S2 into two bands, which are separated along the momentum axis by $\Delta k_{/ /}=0.045 \pm 0.005 \AA^{-1}$.

which was obtained with a photon energy of $26 \mathrm{eV}$ around $\bar{\Gamma}_{2}$ along $\bar{\Gamma} \bar{M} \bar{\Gamma}$. To further enhance the visibility of the splitting of S2, we display, in Figure $3 b$, the second derivative along the energy axis of the ARPES data in Figure 3a. As discussed in the literature, high- $Z$ surface alloys on noble metal surfaces show a Rashba-type of spin splitting looking quite similar to the split of S2. The smallest, barely observable, splitting was found on the $\mathrm{Ag}_{2} \mathrm{Sb}$ surface alloy on $\mathrm{Ag}(111) .^{5}$ Because the atomic number of $\mathrm{Te}$ is increased one step compared to $\mathrm{Sb}$, one can expect to observe a spin splitting as is reported here for the AgTe honeycomb layer. The separation in momentum space of the two sub-bands of S2 for the AgTe honeycomb structure is $\Delta k_{/ /}=0.045 \pm 0.005 \AA^{-1}$. This value is quite similar to the value of $\Delta k_{/ /}=0.06 \AA^{-1}$ for $\mathrm{Ag}_{2} \mathrm{~Pb}^{11}$ but significantly smaller than $\Delta k_{/ /}=0.26 \AA^{-1}$ for the $\mathrm{Ag}_{2} \mathrm{Bi}^{4}$ surface alloy, which shows the largest splitting. In this context, one can note that the prototypical case of a Rashba split, i.e., the Shockley surface state on $\mathrm{Au}(111),{ }^{12,13}$ shows a split of $0.024 \AA^{-1}$.

Based on the number of bands, the periodicity in $k_{/ /}$space, and the line shape of the Te $4 \mathrm{~d}$ spectrum, some more or less likely models can be envisioned. We have considered four models with a $\sqrt{3} \times \sqrt{3}$ supercell with respect to $\operatorname{Ag}(111)$. (i) Te adatoms (1/3 ML) positioned at hollow sites on the $\mathrm{Ag}(111)$ surface. (ii) A monolayer of graphene-like Te, i.e., tellurene. (iii) A substitutional $\mathrm{Ag}_{2} \mathrm{Te}$ surface alloy, i.e., one out of three $\mathrm{Ag}$ atoms in the $\sqrt{3} \times \sqrt{3}$ cell is substituted by Te in the upper atomic layer. (iv) An AgTe 2D binary honeycomb structure. These four models are visualized after full relaxation in Figure S1. The theoretical band structures of all four models are presented together with the experimental one in Figure S2. We find that only model iv can explain all the features observed in the ARPES data.

In the following, we present band structure calculations, which were performed based on DFT using the PAW method $^{14}$ implemented in VASP. ${ }^{15}$ We employed the generalized gradient approximation (GGA) with the $\mathrm{PBE}^{16}$ exchange-correlation. Our calculated results lead to the conclusion that the AgTe layer is well-described by model iv mentioned above and shown in Figure 4a. One Te atom (blue
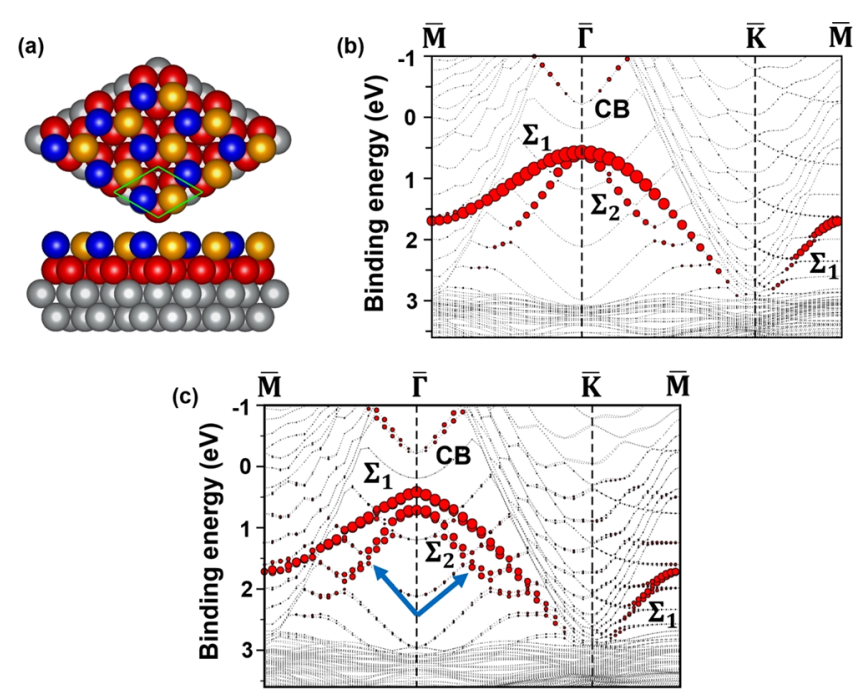

Figure 4. Atomic model and band structure of the AgTe honeycomb structure on $\mathrm{Ag}(111)$ calculated using DFT+PBE. (a) Top and side views of the atomic model. The blue balls represent Te atoms. Yellow balls represent Ag atoms which are part of the honeycomb layer. Red balls represent the first layer of the $\operatorname{Ag}(111)$ slab. Gray balls represent deeper Ag layers. (b and c) The calculated band structure plotted along $\bar{M} \bar{\Gamma} \bar{K} \bar{M}$, without and with spin-orbit coupling, respectively. Two valence bands from the $2 \mathrm{D}$ AgTe layer are labeled $\Sigma_{1}$ and $\Sigma_{2}$, and the conduction band is labeled $\mathrm{CB}$. The blue arrows point at regions where the $\Sigma_{2}$ band shows a small split.

ball) and one $\mathrm{Ag}$ atom (yellow ball) form a honeycomb structure with a lattice constant of $5.0 \AA$, which matches a $\sqrt{3}$ $\times \sqrt{ } 3$ supercell of the $\operatorname{Ag}(111)$ surface. After full relaxation of the slab, described in Methods, the AgTe honeycomb structure was stable on the $\operatorname{Ag}(111)$ surface with very small buckling ( $\mathrm{Te}$ atoms being $0.1 \AA$ higher than Ag atoms). Panels $\mathrm{b}$ and $\mathrm{c}$ of Figure 4 show the results of band structure calculations, plotted along $\bar{M} \bar{\Gamma} \bar{K} \bar{M}$, without and with spin-orbit coupling, respectively. Red dots highlight $\mathrm{p}_{x} / \mathrm{p}_{y}$ states of the AgTe layer. Comparing Figures 2 and 4, one finds a striking agreement between the ARPES data and the calculated band structure with spin-orbit coupling. The calculated $\Sigma_{1}$ and $\Sigma_{2}$ bands in Figure $4 c$ reproduce the dispersions of $S 1$ and $S 2$ extremely well. There is an energy difference of $\sim 0.31 \mathrm{eV}$ between $\Sigma_{1}$ and 
$\Sigma_{2}$ at the $\bar{\Gamma}$ point, which closely reproduces the experimental separation of $\sim 0.36 \mathrm{eV}$ between $\mathrm{S} 1$ and S2. Regarding the absolute energy positions of $\Sigma_{1}$ and $\Sigma_{2}$ compared to S1 and S2, we find a difference of $\sim 0.3 \mathrm{eV}$. A most likely reason for the deviation is that self-energy effects, to obtain the quasiparticle band structure, are not included in the present calculations. ${ }^{17}$ Another extraordinary agreement is the small but resolvable splitting of $\Sigma_{2}$, with $\Delta k_{/ /} \approx 0.040 \AA^{-1}$, which compares closely to the experimental splitting of $\Delta k_{/ /} \approx 0.045 \AA^{-1}$. The combination of spin-orbit coupling and the broken inversion symmetry at the surface point to a split of the Rashba type of $\Sigma_{2} /$ S2. Such spin-splits have been extensively studied in the case of substitutional surface alloys, such as $\mathrm{Ag}_{2} \mathrm{~Pb}$ and $\mathrm{Ag}_{2} \mathrm{Bi}^{4,11}$ It is interesting to note that the calculated value of the direct band gap at $\bar{\Gamma}$ in Figure $4 \mathrm{c}$ is $\sim 0.65 \mathrm{eV}$, which is very close to the $\sim 0.75 \mathrm{eV}$ binding energy of $\mathrm{S} 1$ in Figure 2. This indicates that the Fermi level of the system is actually pinned at an energy very close to the conduction band minimum located at $\bar{\Gamma}$.

A theoretical band structure including spin orbit coupling was presented along the $\bar{\Gamma} \overline{\mathrm{K}}$ line for a honeycomb model of AgTe in ref 10 . That band structure is very similar to the $\bar{\Gamma} \overline{\mathrm{K}}$ part of Figure $4 \mathrm{c}$ except that the splitting in the lower band was not identified. The calculated band structure was compared to ARPES data along $\bar{\Gamma} \overline{\mathrm{K}}$. However, the broad ARPES data could not resolve the two bands, $\Sigma 1$ and $\Sigma 2$, predicted by theory. Thus, the comparison with the theoretical band structure did not provide conclusive evidence to confirm the formation of a AgTe honeycomb structure in ref 10 . Our detailed highresolution ARPES data and the sharp LEED pattern from the AgTe layer provide sufficient information to verify the existence of an undistorted AgTe honeycomb layer on the $\operatorname{Ag}(111)$ substrate.

In summary, we have successfully grown a $2 \mathrm{D}$ binary AgTe honeycomb structure on $\operatorname{Ag}(111)$. The atomic and electronic structures were experimentally examined by LEED, core-level spectroscopy, and ARPES. The high-resolution ARPES data along the two major symmetry lines showed sufficient details to make a conclusive comparison to the calculated band structure. Not only the dispersions of the two bands (S1 and S2) but also the spin-split of the S2 band were closely reproduced by the model calculations. These results, together with the sharp LEED pattern, make a strong case for the formation of an undistorted AgTe honeycomb layer. Our results on this new type of $2 \mathrm{D}$ material should inspire further investigations on honeycomb structured binary alloys.

\section{METHODS}

Samples were prepared in situ in an ultrahigh vacuum (UHV) system equipped with LEED and ARPES instruments. ARPES and core-level data were obtained at beamline I4 at the MAXlab synchrotron radiation facility. The data were acquired at room temperature by a Phoibos 100 analyzer from Specs with a two-dimensional detector. The energy and angular resolutions were $50 \mathrm{meV}$ and $0.3^{\circ}$, respectively. First-principles density functional theory (DFT) calculations were used to interpret the experimental electronic structure data from ARPES. Atomic structures were modeled by a slab, which was built from nine Ag layers terminated by a Te containing $\sqrt{ } 3 \times \sqrt{3}$ supercell. Four different structures were considered (see Figure S1). About $19 \AA$ of vacuum spacing was used to avoid interaction between neighboring slabs of the periodic structure. The positions of all atoms were fully relaxed using the functional of Perdew, Burke, and Ernzerhof (PBE) and the projector augmented wave (PAW) method including van der Waals (vdW) interaction within the Vienna ab initio simulation package (VASP) code. The energy cutoff of the plane-wave basis set was $375 \mathrm{eV}$, and the k-point mesh was (9 $\times 9 \times 1)$. All atoms were relaxed until the average force was within $0.01 \mathrm{eV} / \AA ̊$. Band structure calculations were implemented considering spin-orbital coupling.

\section{ASSOCIATED CONTENT}

\section{Supporting Information}

The Supporting Information is available free of charge at https://pubs.acs.org/doi/10.1021/acs.jpclett.0c00123.

Atomic models referred to in the Letter, theoretical band structures of each model calculated using DFT, and comparisons to the experimental band structure obtained by ARPES (PDF)

\section{AUTHOR INFORMATION}

\section{Corresponding Author}

W. Wang - Department of Physics, Chemistry, and Biology, Linköping University S-581 83 Linköping, Sweden; 다이.org/0000-0002-1036-070X; Email: weimin.wang@ maxiv.lu.se

\section{Authors}

J. Shah - Department of Physics, Chemistry, and Biology, Linköping University S-581 83 Linköping, Sweden

H. M. Sohail - Department of Physics, Chemistry, and Biology, Linköping University S-581 83 Linköping, Sweden

R. I. G. Uhrberg - Department of Physics, Chemistry, and Biology, Linköping University S-581 83 Linköping, Sweden

Complete contact information is available at:

https://pubs.acs.org/10.1021/acs.jpclett.0c00123

\section{Author Contributions}

J.S. conducted the experiments together with R.I.G.U and H.M.S. W.W. did the theoretical modeling and wrote the Letter together with R.I.G.U and J.S. All authors took part in data analysis and discussions of the Letter before submission. R.I.G.U. is the group leader.

\section{Notes}

The authors declare no competing financial interest.

\section{ACKNOWLEDGMENTS}

Technical support from Dr. Johan Adell, Dr. Craig Polley, and Dr. T. Balasubramanian at MAX-lab is gratefully acknowledged. Financial support was provided by the Swedish Research Council (Contract No. 621-2014-4764). The calculations were carried out at the National Supercomputer Centre (NSC) supported by the Swedish National Infrastructure for Computing (SNIC).

\section{REFERENCES}

(1) Geim, A. K.; Novoselov, K. S. The Rise of Graphene. Nat. Mater. 2007, 6, 183.

(2) Krawiec, M. Fuctionalization of Group-14 Two-Dimensional Materials. J. Phys.: Condens. Matter 2018, 30, 233003 and references therein..

(3) Bychkov, Y. A.; Rashba, E. I. Properties of a 2D Electron Gas with Lifted Spectral Degeneracy. JETP Lett. 1984, 39, 78. 
(4) Ast, C. R.; Henk, J.; Ernst, A.; Moreschini, L.; Falub, M. C.; Pacilé, D.; Bruno, P.; Kern, K.; Grioni, M. Giant Spin Splitting through Surface Alloying. Phys. Rev. Lett. 2007, 98, 186807.

(5) Moreschini, L.; Bendounan, A.; Gierz, I.; Ast, C. R.; Mirhosseini, H.; Höchst, H.; Kern, K.; Henk, J.; Ernst, A.; Ostanin, S.; et al. Assessing the Atomic Contribution to the Rashba Spin-Orbit Splitting in Surface Alloys: $\mathrm{Sb} / \mathrm{Ag}(111)$. Phys. Rev. B: Condens. Matter Mater. Phys. 2009, 79, 075424.

(6) Shao, Y.; Liu, Z.-L.; Cheng, C.; Wu, X.; Liu, H.; Liu, C.; Wang, J.-O.; Zhu, S.-Y.; Wang, Y.-Q.; Shi, D.-X.; et al. Epitaxial Growth of Flat Antimonene Monolayer: A New Honeycomb Analogue of Graphene. Nano Lett. 2018, 18, 2133.

(7) Lin, X.; Lu, J. C.; Shao, Y.; Zhang, Y. Y.; Wu, X.; Pan, J. B.; Gao, L.; Zhu, S. Y.; Qian, K.; Zhang, Y. F.; et al. Intrinsically Patterned Two-Dimensional Materials for Selective Adsorption of Molecules and Nanoclusters. Nat. Mater. 2017, 16, 717.

(8) Gao, L.; Sun, J.-T.; Lu, J.-C.; Li, H.; Qian, K.; Zhang, S.; Zhang, Y.-Y.; Qian, T.; Ding, H.; Lin, X.; et al. Epitaxial Growth of Honeycomb Monolayer CuSe with Dirac Nodal Line Fermions. Adv. Mater. 2018, 30, 1707055.

(9) Dong, L.; Wang, A.; Li, E.; Wang, Q.; Li, G.; Huan, Q.; Gao, H.J. Formation of Two-Dimensional AgTe Monolayer Atomic Crystal on $\operatorname{Ag}(111)$ Substrate. Chin. Phys. Lett. 2019, 36, 028102.

(10) Liu, B.; Liu, J.; Miao, G.; Xue, S.; Zhang, S.; Liu, L.; Huang, X.; Zhu, X.; Meng, S.; Guo, J.; et al. Flat AgTe Honeycomb Monolayer on $\operatorname{Ag}(111)$. J. Phys. Chem. Lett. 2019, 10, 1866.

(11) Pacilé, D.; Ast, C. R.; Papagno, M.; Da Silva, C.; Moreschini, L.; Falub, M.; Seitsonen, A. P.; Grioni, M. Electronic Structure of an Ordered $\mathrm{Pb} / \mathrm{Ag}(111)$ Surface Alloy: Theory and Experiment. Phys. Rev. B: Condens. Matter Mater. Phys. 2006, 73, 245429.

(12) La Shell, S.; McDougall, B. A.; Jensen, E. Spin Splitting of an $\mathrm{Au}(111)$ Surface State Band Observed with Angle Resolved Photoelectron Spectroscopy. Phys. Rev. Lett. 1996, 77, 3419.

(13) Cercellier, H.; Didiot, C.; Fagot-Revurat, Y.; Kierren, B.; Moreau, L.; Malterre, D.; Reinert, F. Interplay between Structural, Chemical, and Spectroscopic Properties of $\mathrm{Ag} / \mathrm{Au}(111)$ Epitaxial Ultrathin Films: A Way to Tune the Rashba Coupling. Phys. Rev. B: Condens. Matter Mater. Phys. 2006, 73, 195413.

(14) Kresse, G.; Joubert, D. From Ultrasoft Pseudopotentials to the Projector Augmented-Wave Method. Phys. Rev. B: Condens. Matter Mater. Phys. 1999, 59, 1758.

(15) Perdew, J. P.; Burke, K.; Ernzerhof, M. Generalized Gradient Approximation Made Simple. Phys. Rev. Lett. 1996, 77, 3865.

(16) Kresse, G.; Furthmüller, J. Efficient Iterative Schemes for $A b$ Initio Total-Energy Calculations using a Plane-Wave Basis Set. Phys. Rev. B: Condens. Matter Mater. Phys. 1996, 54, 11169.

(17) Aryasetiawan, F.; Gunnarsson, O. The GW Method. Rep. Prog. Phys. 1998, 61, 237. 